\title{
CARDIAC DYSFUNCTION IN CANCER PATIENTS RECEIVING CHEMOTHERAPY
}

\author{
Sivaranjani $H^{1}$, Prashanth V. N2,
}

${ }^{1}$ Assistant Professor, Department of General Medicine, Bangalore Medical College \& Research Institute, Bangalore. ${ }^{2}$ Assistant Professor, Department of General Medicine, Bangalore Medical College \& Research Institute, Bangalore.

ABSTRACT: AIM OF THE STUDY: The objectives of this study were to assess myocardial dysfunction and clinical cardiac events, to compare these features in the 3 groups (CT, RT, CT+RT) and to look for correlation between low LVEF, age, sex, dose of treatment, interval from treatment to testing.

MATERIALS AND METHODS: The subjects of this study were 75 cases of cancer patients between 18-65 years of age, who received anthracycline containing chemotherapy (CT), chest radiotherapy (RT), or both (CT+RT). They were divided into 3 groups with 25 patients in each that is CT, RT, CT + RT groups. These patients did not have diabetes, hypertension, not in peripartum and nonalcoholics. A baseline chest x ray, ECG, echocardiogram were done in these patients before starting them on treatment and a cardiac disease had been ruled out. Type of treatment and dose was also noted. A detailed history and physical examination along with chest $\mathrm{x}$ ray, ECG, 2D Echo was done in all patients. Stress test was done where indicated.

RESULTS: Signs and symptoms of CHF was seen in 9.33\% (7 cases) of patients, out of them, 85.71\% belonged to CT+RT group. Chest $\mathrm{X}$-Ray finding of cardiomegaly was also significant among the 3 groups. $16 \%$ of patients had LVEF $<55 \%$. The majority of them belonged to the CT+RT group. Out of these 58.33\% had borderline systolic dysfunction -LVEF (50-55\%), 41.66\% of them had very low LVEF of $<40 \%$. Valvular lesions were seen in $12 \%$ of the patients. Left ventricular end diastolic diameter was also most significantly increased in the CT+RT group. There was no correlation between low LVEF, age, dose of therapy, sex or interval from therapy to testing.

CONCLUSION: This study shows that only when anthracycline containing chemotherapy and chest radiotherapy are combined, synergistic cardiotoxicity increases than either modality in isolation. There was no significant correlation between age, sex, dose of treatment, interval from treatment to testing and the development of low LVEF. As this study has a limited sample size, it needs to be confirmed with a larger group.

KEYWORDS: Radiotherapy (RT); Chemotherapy (CT); Synergistic cardiotoxicity; Systolic dysfunction; Left ventricular ejection fraction (LVEF); Congestive heart failure; Cardiomegaly; Valvular lesions; Left ventricular end diastolic diameter.

HOW TO CITE THIS ARTICLE: Sivaranjani H, Prashanth V. N. "Cardiac Dysfunction in Cancer Patients Receiving Chemotherapy". Journal of Evolution of Medical and Dental Sciences 2015; Vol. 4, Issue 90, November 09; Page: 15578-15581,

DOI: $10.14260 /$ jemds/2015/2234.

INTRODUCTION: Cancer is the second leading cause of death next to heart disease. The primary goal of cancer treatment is to eradicate cancer and preserve the quality of life. Cancer treatment includes chemotherapy and radiotherapy. These modalities are often used in combination with an intention of causing regression of established tumours or to slow the rate of tumour growth. Although these treatments have selectivity for cancer cells, they produce side effects. Since the five year survival rate of all cancer patients is now $59 \%{ }^{1}$ growing attention now is focused on the adverse effects such as myocardial dysfunction due to cancer therapy, and the prevention and alleviation of the same in the already suffering patients. Due to their broad antineoplastic activity, anthracycline are the most widely used agents in oncological practice. Their efficacy for the treatment of various haemopoetic (Acute leukaemia, malignant lymphoma) or solid tumours (Mainly cancer of breasts, lungs, thyroid, paediatric solid tumours, osteosarcoma)

Financial or Other, Competing Interest: None.

Submission 03-10-2015, Peer Review 05-10-2015,

Acceptance 26-10-2015, Published 09-11-2015.

Corresponding Author:

Dr. Prashanth. V. N

\#9, Karmic Road, Shankarapuram,

Bangalore-560004.

E-mail: drprashaanthvn2000@yahoo.com

DOI:10.14260/jemds/2015/2234. has been well established. The most serious side effect of anthracyclines is cardiotoxicity. ${ }^{2}$ the risk of cumulative, life threatening toxic cardiomyopathy has an effect on the total dose of these drugs and reduces their therapeutic potential. In patients on anthracyclines with or without mediastinal irradiation, clinical or subclinical abnormalities of ventricular function have been documented. During radiotherapy of the mediastinum or of the chest wall as in breast cancer, there is often some irradiation of the heart and major blood vessels, which could increase cardiovascular mortality years later. The prevalence of radiation associated cardiac disease is increasing due to prolonged survival following mediastinal irradiation. Side effects of radiation include pericarditis, accelerated coronary artery disease, myocardial fibrosis, and valvular injury. Studies have shown that compared to the general population, patients on anthracyclines were associated with a three to four fold increase in risk of cardiac events, especially when high dose volumes of cardiac irradiation were also delivered.

\section{AIM OF THE STUDY:}

1. To assess the extent of myocardial dysfunction and clinical cardiac events in cancer patients who have received cancer treatment.

2. To compare the clinical features, chest x-ray, ECG, Echocardiographic findings in the 3 groups of patients who have received Anthracycline containing chemotherapy, chest radiotherapy or both. 
3. To assess the correlation of LVEF with age, sex, dose of the therapy and interval from treatment to testing.

MATERIALS AND METHODS: 75 cases of cancer patients between 18-65years of age who have received cancer treatment in Victoria Hospital, BMC\& RI, Bangalore, at least one year back or more were the subjects of this study. The duration of study was between June 2012 to April 2014. They had received chemotherapy, mediastinal or chest radiotherapy or both and are divided into 3 groups-those who received chemotherapy (CT), those who received radiotherapy (RT) and those who received both (CT + RT) with 25 patients in each of them respectively.

1. Patients were identified from medical records. These were patients who received anthracycline containing chemotherapy, radiotherapy or both. These were patients in whom chest x-ray, ECG, 2D Echo were done before starting treatment and were normal.

2. Type of treatment and total dose regarding cancer treatment (Especially anthracycline dose in case of chemotherapy and radiation dose) were recorded.

3. Detailed history of symptoms referring to cardiovascular system such as exertional dyspnoea, orthopnoea, paroxysmal nocturnal dyspnoea, night cough, effort intolerance, pedal edema, were recorded.

4. Detailed physical examination with special reference to cardiovascular system like tachycardia, pulsus alternans, raised jugular venous pressure, gallop rhythm, basal crepitations, tender hepatomegaly were noted.

5. 12 lead electrocardiogram was done in all patients. Changes like loss of voltage in $\mathrm{R}$ wave, sinus tachycardia, prolongation of QT interval, flattening of $\mathrm{T}$ wave, ventricular ectopic activity, right bundle branch block were noted.

6. 2D Echocardiogram was done in all patients. LVEF was calculated according to Teiccholz AE formula in patients with normal ventricles or in those with diffuse hypokinesia. Values of $\mathrm{LVEF}<50 \%$ were considered clearly abnormal, whereas values between $50 \%$ and $55 \%$ were classified as borderline. The definition of systolic dysfunction was applied to all cases with abnormal and borderline LVEF. Left ventricular enlargement is defined as an end-diastolic diameter exceeding $3.2 \mathrm{~cm} / \mathrm{m}^{2}$ of body surface area. ${ }^{2}$ Diastolic dysfunction, global hypokinesia, pericardial effusion and valvular lesions such as mitral and aortic regurgitation were also noted.

7. Exercise Stress Test was done only where indicated.

8. All patients were included only after receiving a valid informed written consent

9. Ethical committee clearance was taken before the study.

\section{Inclusion Criteria:}

1. Cancer patients between 18 and 65 years.

2. Cancer patients who have received anthracycline containing chemotherapy and or mediastinal or breast radiotherapy atleast one year back or more:

\section{Exclusion criteria:}

1. Cancer patients $<18$ years.

2. Cancer patients who are known:

a. Diabetics.

b. Hypertensives.

c. Chronic alcoholics.

d. In peripartum.

e. To have pre-existing cardiac disease.

STATISTICAL ANALYSIS: SPSS statistical software was used to perform the analysis. Pearsons chi square test was used to find significant difference between the 3 groups in developing the various clinical features and investigational findings. The likelihood ratio and linear by linear association between the various factors was also found. A p value $<0.005$ was considered statistically significant. Multivariate analysis of the potential effect of factors such as age, sex, dose of treatment and interval from treatment to testing on the development of low LVEF was made using logistic regression.

\section{RESULTS:}

- A cohort of 75 patients was identified and were divided into 3 groups with 25 patients in chemotherapy (CT) group, 25 patients in radiotherapy (RT) group and 25 patients in chemotherapy+ radiotherapy $(\mathrm{CT}+\mathrm{RT})$ group.

- The mean age of patients was 46 years 6 months with a range of 22 to 64 years.

- Maximum number of patients belonged to the $41-50$ years age group followed by those of $51-60$ years.

- Males were $26.6 \%$ while females were $73.3 \%$.

- Mean duration since diagnosis was 2 years 6 months with a range 1 to 8 years.

- In the CT group $68 \%$ had non-Hodgkin's lymphoma and $32 \%$ had Hodgkin's lymphoma. In the RT group $80 \%$ had breast cancer and $20 \%$ had oesophageal cancer. In the CT+RT group $96 \%$ of them had breast cancer and $4 \%$ had non-Hodgkin's lymphoma.

- $\quad$ Among the RT and CT + RT groups combined, $44 \%$ had received left chest radiotherapy, $44 \%$ had received right chest radiotherapy and $12 \%$ had received mediastinal irradiation.

- $\quad 7$ Patients (9.33\%) developed one or the other signs and symptoms of congestive heart failure, out of which 1 patient (14.28\%) belonged to the CT group and6 patients (85.71\%) belonged to CT+RT group. There is a statistically significant difference in the development of signs and symptoms in the 3 groups with the CT+RT group having the most significant association with the signs and symptoms $(\mathrm{p}<0.008)$.

- $\quad$ There is a significant difference in the chest $x$-ray features suggestive of cardiomegaly and pulmonary oedema among the 3 groups with the $\mathrm{CT}+\mathrm{RT}$ group having the most significant association $(\mathrm{p}<0.008)$.

- $\quad$ The ECG findings were not statistically significant in the 3 groups.

- 12 patients $(16 \%)$ developed $L V E F<55 \%$ with significant difference in the 3 groups. $40 \%$ (10) of the patients in the CT+RT group had LVEF $<55 \%$ with the most significant association $(\mathrm{p}<0.000) .1$ patient $(4 \%)$ each in the CT and RT group had a low EF. Out of the 12 patients $58.33 \%$ of 
them had borderline systolic dysfunction and $41.66 \%$ of them had $\mathrm{LVEF}<40 \%$. The CT+RT group had maximum number of patients with $\mathrm{LVEF}<40 \%$.

- Among the patients who received radiotherapy (RT \& CT+RT groups) $22 \%$ (11 patients) had systolic dysfunction, out of these $6(54.5 \%)$ patients had received left sided radiotherapy to the chest and 5 $(45.5 \%)$ patients had received right sided radiotherapy.

- $14.66 \%$ of the patients had diastolic dysfunction but this was not statistically significant in the 3 groups.

- There was no statistically significant difference in the development of global hypokinesia on 2D Echo in the 3 groups.

- Only 2 patients (4\%) developed mild pericardial effusion belonging one each to RT and CT+RT groups which was not statistically significant.

- $12 \%$ of the patients developed valvular lesions with a tendency to have significant difference in the 3 groups with CT+RT group having the most significant chances of developing valvular lesions $(\mathrm{p}<0.011)$.

- Left ventricular end diastolic diameter was also found to be significantly different in the 3 groups with again the CT+RT group being most prone to develop an enlarged left ventricle $(\mathrm{p}<0.001)$.

- However there is no correlation between age, sex, interval from treatment to testing or dose (radiotherapy and anthracycline chemotherapy) and LVEF in the study.

DISCUSSION: There is very limited Indian data regarding long term cardiac effects of treatment in patients with solid tumours. This study was very useful in providing baseline data and also direction for further research in this matter. Our study consisted of a cohort of 75 patients who were divided into 3 groups with 25 patients each in CT, RT and $\mathrm{CT}+\mathrm{RT}$ groups. The youngest patient in the study was 22 years old and the oldest was 64 years with mean age of the patients being 46 years 6 months. In this study the maximum number of patients belonged to the 41-50 years age group. The male to female ratio was $1: 2.75$. The mean interval from treatment to testing was 2 years 6 months with the range being 1-8 years.

There were $68 \%$ cases of NHL and $32 \%$ cases of HD in the CT group. In the RT group $80 \%$ had breast cancer and $20 \%$ had oesophageal cancer. In the CT + RT group, $96 \%$ had breast cancer and $4 \%$ had NHL. In the patients who received radiotherapy (RT and $\mathrm{CT}+\mathrm{RT}$ groups) equal numbers of patients received left and right chest radiotherapy (44\% each), while the remaining $12 \%$ received mediastinal radiotherapy.

This study showed that $9.33 \%$ of the patients of the three groups had experienced one or the other signs and symptoms of CHF and only one patient out of them had received CT whereas the remaining 6 patients $(85.71 \%)$ had received CT+RT. The patients who received only radiotherapy were asymptomatic. This shows that only when both forms of treatment are combined, synergistic cardiotoxicity increases. In a study by L.J. Steinherz et.al ${ }^{1} 4 \%$ of the patients had late symptoms of cardiac failure. The most common signs and symptoms of my study were exertional dyspnoea, effort intolerance and basal crepitations, however none of the patients had night cough, gallop rhythm or pulsus alternans.

Cardiomegaly on chest $\mathrm{x}$ ray was again seen in maximum number of patients belonging to $\mathrm{CT}+\mathrm{RT}$ group suggesting that combination of chemotherapy and radiotherapy increased the chances of developing cardiomegaly, whereas pulmonary oedema on chest $\mathrm{x}$-ray did not show any statistically significant association in the three groups. An extensive search made for a similar result in other studies did not yield any information.

In this study the various ECG findings were sinus tachycardia, poor $\mathrm{R}$ wave progression, ventricular premature complexes and right bundle branch block. But these were not statistically significant implying that these changes are not specific for anthracycline induced or radiotherapy induced cardiomyopathy. Hence physicians should not depend on them to establish the diagnosis. Instead it can be used in conjunction with echocardiography. ${ }^{2}$

Out of the 75 patients, $16 \%$ developed systolic dysfunction with a LVEF $<55 \% .40 \%$ of patients in CT+RT group had developed significant systolic dysfunction, while only one patient (4\%) each in the CT and RT group had systolic dysfunction. It implies that when combination of anthracycline containing chemotherapy and radiotherapy are given it has a greater association with cardiotoxicity than either modality in isolation. It is believed that structural damage of the heart is connected with the accumulation of adverse effects of chemotherapy and radiotherapy on myocardium.

Anthracyclines principally damage myocytes directly and its ultimate result is diffuse myocardial fibrosis. Radiation injures the endothelium of myocardial capillaries leading to ischemia and myocardial fibrosis. Long term studies in survivors of childhood cancers and breast cancer have confirmed that combined therapy affects left ventricular systolic function and mortality more significantly than either alone.3,4,5 Out of the 12 patients with systolic dysfunctions $58.33 \%$ had borderline LVEF (50-55\%) and $41.66 \%$ had LVEF $<40 \%$. The $\mathrm{CT}+\mathrm{RT}$ group had the maximum number of patients with LVEF $<40 \%$ as well as those with borderline LVEF.

In the 50 patients who had received RT and CT, 6 patients $(55.4 \%)$ had received the right side chest radiotherapy. There is a minimal difference in these two sets of patients which is not significant and is difficult to comment as the number of patients is too low. In this study none of the patients in the RT group had a $\mathrm{LVEF}<50 \%$, implying that the interval from treatment to testing was not long enough to cause myocardial dysfunction as told by earlier studies. It may also be attributable to the cardiac volume irradiated or modern techniques of radiotherapy administration or may be due to improved cardiac function as time from radiotherapy elapses. ${ }^{6}$ Hence long term follow-up is required to study the effects of radiotherapy alone on the myocardium. $14.66 \%$ of the patients belonging to the 3 groups had diastolic dysfunction which was significantly different among the 3 groups.

Similarly global hupokinesia echocardiogram was also not significantly different in the 3 groups. The limited size pf this study underscores the need for larger perspective studies to establish the value of diastolic dysfunction and global hypokinesia in the detection of CT and RT induced 
cardiotoxicity. Left ventricular end diastolic diameter was also found to be significantly different in the 3 groups with again the CT+RT group being most prone for dilated left ventricle as seen in earlier studies.

Two patients (4\%) developed mild pericardial effusion and they belonged one each to RT and CT+RT group, which was not stastically significant. This result can be again due to modern techniques used for giving radiotherapy. In this study $12 \%$ (16 patients) developed a tendency towards significant valvular lesions with again the CT+RT group having the most significant number which is similar to a study by Glanzmann $\mathrm{C}$ et al. ${ }^{7}$

Stress test was done in all patients with LVEF $\leq 55 \%$ and was negative for provocable ischemia indicating that none of these patients had findings due to myocardial ischemia. In this study there was no correlation between low LVEF and various parameters like age, sex, interval from treatment to testing or dose of the treatment. This again could be due to the limited study size. A similar result was seen in a study by Constine L S et al. ${ }^{8}$

\section{Strategies to reduce cardiotoxicity in these patients as} analysed by clinical trials:

1. Observing allowed total dose of anthracycline antibiotics.

2. Modification of total doses in relation to risk factors of cardiotoxicity.

3. Modification of the methods of anthracycline administration:

- 48-96 hours infusion.

- Administration of low doxorubicin doses every week, maintaining total dose.

- Use of drugs in the form of liposome preparations.

4. Administration of synthetic anthracycline analogues characterised by lower cardiotoxicity.

- Idarubicin.

- Mitoxantrone.

5. Pharmacological cardioprotection.

- Cardioxan (dexrazoxane, ICRF-187).

- Vitamin E.

- $\quad \mathrm{N}$-acetylcysteine.

- Prednisone.

- Q-10 coenzyme.

- Digitalis glycoside.

- Probucol.

- Calcium channel blockers.

- Beta-adrenergic receptor blockers.
- Anti-histamine agents.

- Anti-inflammatory agents.

- Carnitine.

- Ascorbic acid

- $\quad$ Bipiridine derivatives (amrinone, milrinone).

6. Individual adjustment of anthracycline dose on the basis of their factual cardiotoxicity and the results of repeated examinations of myocardial function and/or structure.

\section{CONCLUSIONS:}

- This study shows that only when anthracycline containing chemotherapy and chest radiography are combined, synergistic cardiotoxicity increases than either modality in isolation.

- There is no significant correlation between age, sex, dose of the treatment, interval from treatment to testing and the development of low LVEF. As this study has a limited sample size it needs to be confirmed with a larger group.

\section{REFERENCES:}

1. L.J.Steinherz, P.G.Steinherz, C.T.Tan, G.Heller and M.L.Murphy. Cardiac toxicity 4 to 20 years after completing anthracycline therapy. JAMA 1996;266:12.

2. Ganz WI, Sridhar KS, Ganz SS, Gonzalez R, Chakko S, Serafini A. Review of tests for monitoring doxorubicininduced cardiomyopathy. Oncology 1996;53:461-470.

3. Pihkala J, Saarinen U, Lundstorm U. Myocardial function in children and adolescents after therapy with anthracyclines and chest irradiation. Eur J Cancer 1996;32A:97-103.

4. Glanzmann C, Kaufmann P, Jenni R, Hess OM, Huguenin P. Cardiac risk after mediastinal irradiation for Hodgkin's disease. Radiother Oncol 1998;46(1):51-62.

5. Pihkala J, Saarinen UM, Lundstorm U, Virtanen K, Virkola K, Siimes MA, Pesonen E. Myocardial function in children and adolescents after therapy with anthracyclines and chest irradiation. Eur J Cancer 1996;32A(1):97-109.

6. Kesavan Shan, MD; A Michael Lincoff, MD; and James B. Young. Anthracycline induced cardiotoxicity. Ann Intern Med 1996;125:47-58.

7. Glanzmann C, Huguenin P, Lutolf UM. Cardiac lesions after mediastinal radiation for Hodgkin's disease. Radiother Oncol 1994;30:43-54.

8. Constine LS, Schwartz RG, Savage D. Cardiac function, perfusion, and morbidity in irradiated long term survivors of Hodgkin's disease. Int J Radiat Oncol Biol Phys 1997;39:897-906. 